

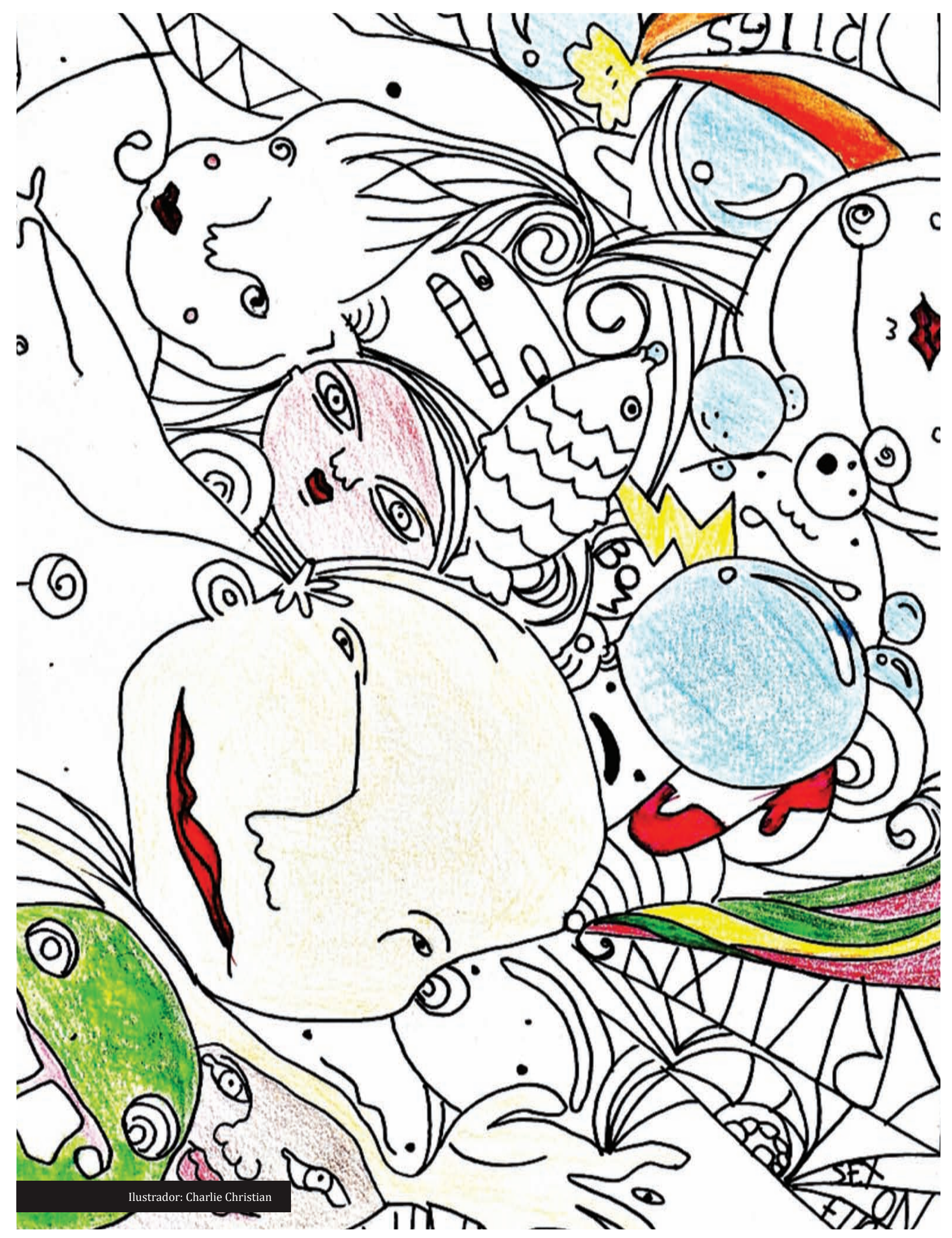




\section{Equipo docente: formación, cultura colaborativa e interdisciplinariedad*}

\author{
Equipe de professores: formação, \\ cultura colaborativa \\ e interdisciplinaridade
}

\author{
Team teaching: training, \\ collaborative culture and \\ interdisciplinarity
}

\section{Resumen}

El equipo docente es una estrategia de trabajo colaborativo que se propone como alternativa de construcción de prácticas pedagógicas en las Escuelas Normales Superiores. Este artículo presenta un recorrido por sus concepciones y propuestas, dando paso a algunas consideraciones sobre su implementación y funcionamiento.

\section{Palabras clave}

Formación de maestros, equipo docente, trabajo colaborativo, Escuela Normal Superior.

\section{Resumo}

A equipe de professores é uma estratégia de trabalho colaborativo que se propõe como uma alternativa na construção de práticas pedagógicas nas Escolas Normais Superiores. Neste artigo apresenta-se um percurso pelas suas concepções e propostas, oferecendo algumas reflexões acerca da sua implementação e funcionamento.

\section{Palavras-chave}

Formação de professores, equipe de professores, trabalho colaborativo, Escola Normal Superior.

\section{Abstract}

Team teaching is a collaborative work strategy proposed as an alternative for the construction of pedagogical practices at the Superior Normal Schools. This paper presents a route around its ideas and proposals, giving way to some considerations about its implementation and functioning.

\section{Keywords}

Teacher's training, team teaching, collaborative work, Superior Normal School.

\section{Hilda Mar Rodríguez Gómez** \\ Jesús Alberto Echeverri Sánchez***}

* Este artículo deriva sus reflexiones del proyecto de investigación "Equipo docente: formación, cultura colaborativa e interdisciplinariedad", financiado por el Comité para el Desarrollo de la Investigación (CODI) de la Universidad de Antioquia, que contó con la participación de investigadores de escuelas normales superiores del departamento de Antioquia, así: Marleny Alzate y Gildardo Castaño, Escuela Normal Superior Rafael María Giraldo (Marinilla); Billián Jiménez, Nancy Montoya y Gilma Luz Marín, Escuela Normal Superior de Envigado; Paula Martínez y Clara Nury Vásquez, Escuela Normal Superior de Medellín; Margarita Rosa Luján y Carlos Jovany Londoño, Escuela Normal Superior Santa Teresita (Sopetrán). El proyecto se desarrolló entre agosto de 2006 y diciembre de 2012.

** Profesora del Departamento de Pedagogía, Facultad de Educación, Universidad de Antioquia, Medellín, Colombia.

*** Profesor de la Facultad de Educación de la Universidad de Antioquia, coordinador nacional del Grupo de Historia de la Práctica Pedagógica, Medellín, Colombia.
Fecha de recepción: 08 de agosto de 2013

Fecha de aprobación: 29 de noviembre de 2013

Pedagogía y Saberes No. 39 Universidad Pedagógica Nacional Facultad de Educación. 2013, pp. 11-20 
L a realización de la investigación Aciforma ${ }^{1}$ dejó en claro la necesidad de formar un sujeto colectivo en las instituciones formadoras de maestros, que para efectos metodológicos y conceptuales se denominó equipo docente. Los alcances de esta categoría resultan de capital importancia no solo para la conceptualización, sino también para la construcción de estrategias para la operacionalización de dicho equipo, de forma tal que sea posible conocer el impacto de su constitución en la configuración del espacio y el tiempo escolares.

En el marco de esas elaboraciones iniciales, el proyecto "Equipo docente: formación, cultura colaborativa e interdisciplinariedad" nos permitió conocer niveles de desarrollo y existencia de equipos docentes en escuelas normales del departamento de Antioquia (Marinilla, Envigado, Antioqueña y Sopetrán). Su naturaleza e intenciones reafirman algunos hallazgos de otros procesos de indagación que hemos llevado a cabo respecto de la formación de maestros ${ }^{2}$, por cuanto se trabaja la singularidad de las instituciones formadoras como dotadas de naturaleza pedagógica, lo que significa, ante todo, capacidad para acumular saber pedagógico y recontextualizarlo, al tiempo que la elaboración teórica y experimental de una propuesta abierta y contemporánea que se plantea desde el campo conceptual y narrativo de la

1 “Apropiación pedagógica del campo intelectual de la educación para la construcción de un modelo comprensivo para la formación de docentes", proyecto de investigación financiado por Colciencias, la Universidad de Antioquia y la Secretaría de Educación y Cultura de Antioquia, entre 2000 y 2003, bajo la dirección del profesor Jesús Alberto Echeverri, que trabajó con los maestros de las escuelas normales superiores de Antioquia en una propuesta de formación basada en lo que se denominó dispositivo formativo comprensivo.

2 Véase, por ejemplo, "Emergencia y consolidación del dispositivo formativo comprensivo en las Escuelas Normales Superiores: su incidencia en la vida cotidiana", financiado por el CODI y la Universidad de Antioquia, coordinado por Alberto Echeverri y realizado entre 2002 y 2005; su objetivo central fue: "Analizar las continuidades, discontinuidades y solapamientos de prácticas, nociones y metodologías tradicionales en las escuelas normales superiores, con respecto a la apropiación de conceptualizaciones pedagógicas y didácticas, estrategias de investigación en el aula y procesos de formación planteados por el dispositivo formativo comprensivo". También se puede consultar el proyecto "Contribución a la formación de un campo conceptual de la pedagogía para la formación de maestros en Colombia", financiado por el CODI y la Facultad de Educación de la Universidad de Antioquia, cuyo objetivo es contribuir a la producción del campo conceptual de la pedagogía para la formación de maestros que permita establecer la comunicación y las fronteras entre los conceptos, los paradigmas y las diferentes culturas pedagógicas apropiadas en Colombia entre 1980-2000. pedagogía ${ }^{3}$ y recoge críticamente tanto la apropiación de la pedagogía universal como las experiencias y experimentaciones vividas durante años en las escuelas normales superiores.

Este proyecto estuvo orientado por preguntas acerca de la naturaleza del equipo docente, por las distinciones con otras formas de organización colectivas y su relación con la construcción del saber. También nos preguntamos: ¿es el equipo docente un concepto con potencia para aglutinar a su alrededor el devenir pedagógico de la escuela normal superior? ¿Qué tradiciones existen en el panorama de la pedagogía contemporánea acerca del equipo docente? ¿De qué manera las relaciones al interior de los núcleos de saber y entre los núcleos contribuyen al fortalecimiento y desarrollo del equipo docente? ¿La construcción teórica y conceptual del equipo docente es la base para su operatividad en las escuelas normales?

\section{Modelos de colaboración docente}

La gama de modelos de colaboración docente es muy diversa en cuanto a orientaciones que los guían e intereses a los que sirven. Sin embargo, se pueden entender como complementarios, por cuanto promueven el intercambio académico, la recuperación de la voz de maestros y maestras. Entre ellos encontramos: grupos de discusión, comunidades de aprendizaje, comunidades académicas, grupos de apoyo, redes de centros, equipos docentes. Veamos.

3 El campo conceptual y narrativo de la pedagogía alude a una espacialidad coartada por una normatividad jurídica, en lucha entre agrupamientos intelectuales e investigativos. Ese campo no se puede asemejar al espacio que circunda la relación entre el maestro y el alumno y que comúnmente se denomina aula, aunque no sobra advertir que nunca la descarta. Tampoco se puede asemejar el campo al espacio que constituye el patio de recreo, ni mucho menos al espacio físico que configura la sala de profesores. Digamos que el espacio que recorre y el recorrido por el campo carecen de porterías y ventanas, para indicarnos que en su constitución no hay oposición entre el adentro y el afuera, sino tensiones que generan discontinuidades y vacíos. A diferencia de la escuela normal superior este campo no está definido a priori, se engendra y existe en el juego de relaciones de fuerzas que lo envuelven. Posee dos atributos, plural y abierto. A diferencia de los programas escolares que seleccionan un conocimiento específico y lo institucionalizan un campo conceptual y narrativo admite la coexistencia de tendencias, escuelas, y corrientes de pensamiento opuestas, su lema es "todo juega", en contraposición al lema "todo vale". El campo es conceptual, pero en ningún momento excluye las luchar por el poder, ya sea en el saber o en lo social. Aún más, él se confunde con la ciudad y no pierde su singularidad, se entrelaza con lo virtual y no pierde su materialidad, y se funda con los relatos sin perder su producción conceptual y sin privarse de ella (Echeverri, 2008, pp.117-118). 


\section{Grupos de discusión}

Estrategia de investigación social que busca recuperar la voz de los participantes a través de la enunciación de las experiencias propias, que se ponen en relación con otros. Se trata de una técnica propia de la psicología y la dinámica de grupos.

\section{Comunidades de aprendizaje}

Colectivos de acción conformados por actores de la educación cuyo propósito es la transformación de las escuelas, mediante el diseño y la puesta en práctica de un proyecto de cambio que tiene en cuenta las condiciones del entorno, las actuaciones educativas y las teorías que, en otros espacios educativos, han dado los mejores resultados.

\section{Comunidad académica}

Grupo de personas, de diferentes disciplinas, que se unen por la convergencia de potencialidades académicas, por el planteamiento de problemas científicos o sociales, con el propósito de aplicar, aprobar y validar el conocimiento.

\section{Grupo de apoyo entre docentes}

Modalidad de apoyo que funciona en la escuela y que busca la resolución de problemas que surgen en la cotidianidad escolar mediante el análisis, el estudio y el seguimiento -colaborativo- de las situaciones que proponen los docentes.

\section{Equipo docente}

Surge como una propuesta de organización de los profesores en las escuelas normales superiores, extensible a otras instituciones formadoras de maestros, de modo que a través de ellos la experiencia de sí y del otro haga visible el cuerpo del maestro, su propio rostro, en los procesos de formación inicial y de formación permanente.

El equipo docente es una institución de saber que encarna, en la institución formadora, las comunidades científicas y un campo conceptual y narrativo de la pedagogía bajo la forma de una estructura colegiada y comunicativa. Sus relaciones se extienden hacia la ciencia, lo público y lo curricular. Con respecto a la ciencia, genera la relación de objetos de conocimiento; y en lo que tiene que ver con lo curricular y didáctico, produce los objetos de enseñanza. Es de anotar que en dichos objetos tiene presencia la formatividad, entendida como moldeamiento del alumno-maestro a partir de los múltiples sentidos del mundo contenidos en los objetos de enseñanza.
Para nuestro caso, el equipo docente nos ofrece la posibilidad de establecer vínculos entre sus miembros, además de poner el énfasis en la producción de conocimiento pedagógico a través de, entre otras estrategias, la reflexión colectiva.

El equipo docente es un espacio de múltiples relaciones; entre ellas se destacan:

1. Las relaciones entre diversas ciencias y saberes unidos por afinidades en la construcción de sus objetos, de conocimientos, de métodos y de sus campos de experiencia y experimentación.

2. La necesidad de investigar problemáticas comunes planteadas desde los interrogantes que surgen en la construcción del plan de estudios y de los proyectos de investigación del núcleo. De este modo, el equipo docente no es la suma o superposición de asignaturas, sino la construcción de relaciones que desde necesidades inherentes, tanto a la ciencia como a la didáctica, se plantean entre los saberes enseñados.

3. Con la exterioridad, con lo público.

\section{El equipo docente, el núcleo y el devenir pedagógico de la Escuela Normal Superior}

En las instituciones formadoras de docentes, el equipo docente se concreta mediante los campos aplicados, entendidos como "campos de apropiación y producción" (Echeverri, 1996, p. 101); esto es, como espacios para la constante reflexión, evaluación y autocorrección de experiencias, innovaciones y conceptualizaciones. El campo aplicado, a la vez que dispersa y difunde los sentidos de la formación, reúne las contribuciones de los núcleos en torno a acciones, teorías, nociones y conceptos y las dirige hacia el maestro y el alumno-maestro como sujeto de formación. En este proceso de conjugación de contribuciones, el campo aplicado las reorienta, las encamina hacia directrices formativas, las convierte en condiciones de existencia de la formación de maestros. En este sentido es que el campo aplicado se entiende como plural y abierto, pues recoge las observaciones, las experiencias, los conceptos y las teorías acerca de los saberes, la pedagogía, la ciencia, la cultura, el arte, la estética, y las pone en juego en un espacio de formación propio de la escuela normal superior.

Su función principal es la de ejercer críticamente sobre todos aquellos aspectos que configuran y se generan a partir de la práctica pedagógica. Esto se hace posible a través de la consideración del concepto 
de enseñanza como una práctica de saber que, inscrita en las realizaciones del enseñar, permite la legitimación de las acciones emprendidas, al tiempo que posibilita la recuperación del papel activo del maestro, en el equipo docente, en la construcción del conocimiento pedagógico.

Un aspecto a considerar en el campo aplicado es el restablecimiento de las relaciones entre teoría y práctica, pues de esta resulta un maestro crítico capaz de construir espacios de acción. Se trata, de acuerdo con Giroux, de emplear el concepto de radical de manera amplia y abierta:

\begin{abstract}
El concepto de radical sugiere diversas consideraciones para crear las posibilidades de participación humana y desarrollar espacios públicos organizados en torno a cuestiones de diálogo, justicia social, libertad e igualdad. Desde esta perspectiva, radical denota para mí la suposición de que todas las formas de enseñanza, aprendizaje y conocimiento contribuyen a hacer posible que la gente no sólo entienda el mundo, sino que también actúe en el mundo y lo transforme (1996, p. 238).
\end{abstract}

Este permitirá tomar en cuenta la experiencia del maestro, su subjetividad, como un componente básico en la conjugación de la teoría y práctica. Comprender las formas y modos en que la experiencia del maestro se organiza, regula, valida y se refleja es un punto fundamental al momento de construir un espacio capaz de alojar manifestaciones pedagógicas.

En el caso nuestro, sería posible emplear el término experiencias críticas para aludir a situaciones y sucesos que, sumados y actuados, posibilitan la permanencia del espíritu colectivo en las empresas pedagógicas de la institución; esto es, permite la unidad de disposiciones pedagógicas para el equipo docente, consolidan las bases pedagógicas de la formación de maestros -al presentar las opciones que tiene la enseñanza (como concepto y práctica) al interior de la institución normalista para nuclear y dispersar las fuerzas formativas de la acción del maestro-y ayudan al proceso de estructuración de la subjetividad del maestro, en la medida en que propicia el encuentro consigo mismo a través de la enseñanza y múltiples dispositivos ${ }^{4}$.

Porque la experiencia no es algo que esté dado y desde sí mismo se plantee como acto fundamental y primero, su importancia para la constitución del campo aplicado tiene que ver con su capacidad para construir comprensiones de los acontecimientos, las

4 Un dispositivo pedagógico será, entonces, cualquier lugar en el que se constituye o se transforma la experiencia de sí. Cualquier lugar en el que se aprenden o se modifican las relaciones que el sujeto establece consigo mismo (Larrosa, 1995). cuales luego se pueden volver redes de significación y sentido, mismas que se convierten en el piso común de la vivencia de la normal, de la experiencia del equipo docente. Además, la experiencia tejida y enlazada con otras experiencias, vinculada a la reflexión crítica de esta, conforma la historia de la práctica, de modo que sea posible reconocer aquellas acciones, discursos, miradas y silencios que se han dispersado en la bruma de la innominación. La experiencia y su red son la historia que permite la existencia de un campo abierto para la práctica: el campo aplicado.

La teoría y la práctica, en sus formas conjugadas y operantes, permiten la constitución del campo aplicado, por cuanto ofrecen la estructura de un ejercicio crítico de la producción pedagógica, ofrecen condiciones para la transformación de la institución educativa, ya que a través de esta relación se supera.

De lo que se trata, entonces, en el campo aplicado, y también en el equipo docente, es de retomar la experiencia como el espacio en el que los referentes formativos y los conceptos articuladores producen sentidos y significados que se conjugan e insertan con estructuras móviles, cambiantes y complejas, produciendo en este movimiento un tejido y un espacio en el cual se configura la práctica pedagógica. Esta, en el interior del dispositivo, permite al maestro -por cuanto posibilita su organización en complejos y redes- un mayor desarrollo pedagógico, didáctico y científico, pues lo inscribe en una lógica y una estructura institucional cruzada por el equipo docente y sus tejidos de acción y reflexión.

Así las cosas, el equipo docente establece las condiciones de relación entre el maestro y los demás sujetos de la práctica pedagógica (alumnos y directivos), así como del maestro consigo mismo. En otras palabras, el equipo docente es una vía para para la producción pedagógica, el encuentro entre los docentes y la reflexión. Como dicen Hargreaves et al:

Las culturas basadas en la cooperación y las relaciones colegiales dentro de un mismo centro, y también con otras escuelas, proporcionan apoyos que resultan esenciales para implementar cambios eficaces y posibilitar su mantenimiento a lo largo del tiempo, tal y como hemos observado en nuestro estudio. Gracias a ellas puede realizarse el trabajo emocional e intelectual que el cambio requiere, y al garantizar que los cambios sobreviven a los dos o tres individuos que los han preconizado, permiten que las reformas se sostengan al pasar el tiempo. Las culturas de colaboración en los centros escolares ofrecen un contexto para que se desarrolle una formación eficaz del profesorado, siendo a veces ellas mismas las que la proporcionan. Su creación y perpetuación dependen en buena medida de 
la existencia de un liderazgo escolar cualificado (2001, p. 178).

Además, a partir del equipo docente se pueden construir alternativas diferentes al modelo de la anexa, de modo que la uniformidad de la acción, en la práctica como ejercicio rutinario, se vea alimentada por la multiplicidad de sentidos de la práctica en el contexto de la colectividad, no como suma de sujetos, sino como maestros activos que ocupan lugares de saber y acción.

Además de espacios de relaciones, los núcleos tienen funciones formativas en dos niveles: una autoformativa, por cuanto le permite al maestro transformar su mirada sobre el conocimiento científico en el contacto directo que establece con la ciencia en relación con los objetos de conocimiento, sobre el papel que como intelectual debe asumir en la construcción de objetos de enseñanza. En segunda instancia, el núcleo cumple funciones coformativas, pues la misma estructura organizativa que posee (interdisciplinaria) posibilita el intercambio de situaciones, problemas, temáticas y experiencias en torno a la ciencia, la pedagogía y la didáctica, con lo que la reflexión, el análisis y la discusión se hace colegiadamente.

El equipo docente y los núcleos son redes de trabajo y creación de la práctica, donde se encuentran fundamentos para la teoría y la práctica pedagógicas. Esta dinámica de funcionamiento colectivo en red teje sentidos, acciones, conceptos, nociones y experiencias sobre la formación de maestros, como base de la naturaleza pedagógica de la escuela normal superior.

Los núcleos, entonces, se conciben como el espacio académico que fortalecerá lo colegiado, lo cooperativo y lo autogestionado, mediado por la investigación y en relación con la pedagogía, la ciencia, la cultura y el entorno. Los núcleos, por tanto, son la principal estrategia para la construcción del equipo docente, que funge como vehículo de comunicación con las ciencias y el espacio de posible conceptualización pedagógica y didáctica. En tal sentido, cualquier diálogo propuesto debe realizarse en el marco del fortalecimiento de los núcleos.

En fin, de lo que se trata con el equipo docente es forjar el campo aplicado, como campo de experimentación e investigación pedagógica, "sobre registros que vinculen la escritura a la tradición escrita y ésta a la cotidianidad del maestro y el entorno cultural" (Echeverri, 1996, p. 99). Es un intento de unir enseñanza e investigación a través de la experimentación, como forma de legitimar la naturaleza formativa de la escuela normal superior, pues concebimos que la enseñanza es un oficio que precisa de la reflexión autónoma y la elaboración de pensamiento propio, por lo que los maestros deben constituirse como intelectuales, no como individuos que hacen de su labor una actividad solitaria, sino de quien reconoce que es ineludible e indispensable el trabajo en equipo, cooperativo, colegiado y autogestionario.

Así, el equipo docente y los núcleos son redes de trabajo y creación de la práctica, en donde se encuentran fundamentos para la teoría y la práctica. Esta dinámica de funcionamiento colectivo en red teje sentidos, acciones, conceptos, nociones, experiencias sobre la formación de maestros, como base de la naturaleza pedagógica de la escuela normal superior.

\section{Equipo docente, formación de pares y cultura colaborativa}

En trabajos anteriores, a partir de la reestructuración de las escuelas normales superiores de Colombia ${ }^{5}$, hemos insistido en la conformación del equipo docente como condición de posibilidad de los procesos de formación permanente de los maestros, cuya forma de expresión son los seminarios de núcleos ${ }^{6}$. Hablamos de procesos de formación permanente -mas no de cursos de capacitación intermitentes-, que pueden y deben traducirse en innovaciones educativas significativas, sin omitir las mejoras recurrentes en la dignificación de la profesión docente.

Por ello, introducir en las escuelas normales superiores una cultura basada en la colaboración supone, entonces, un cambio en las estrategias de encuentro y formación, decididas por los intereses colectivos, la organización de grupos de trabajo de carácter inter o transdisciplinario, la adopción de métodos de colaboración habituales entre pares académicos, y de nuevas formas de comunicación y de intercambio de experiencias entre iguales que contribuyan a la promoción de la escritura de los maestros.

El desarrollo de esta cultura de la colaboración propone la existencia de un equipo docente agrupado en torno a proyectos de investigación

5 Nos referimos aquí al proyecto de reestructuración de las escuelas normales que se inició en el departamento de Antioquia en 1995, financiado por la Secretaría de Educación para la Cultura; al proyecto Aciforma, financiado por Colciencias, ejecutado entre 1996 y 2000, al proyecto sobre "Emergencia y consolidación del dispositivo formativo comprensivo en las escuelas normales superiores: su incidencia en la vida cotidiana", financiado por el CODI, entre 2002 y 2005.

6 El seminario de núcleos es un espacio de encuentro entre los maestros en el que se conjugan los saberes, los conceptos, las prácticas, las experiencias, las experimentaciones e innovaciones de las diferentes normales, para luego devolverlo a estas a través de la investigación como forma básica de existencia de la práctica. 
interdisciplinaria, situados en dos dimensiones: 1) la selección de objetos de conocimiento, bajo el supuesto de que los maestros son agentes de un saber específico y mantienen relaciones constantes con las comunidades académicas, o al menos acceden con frecuencia a las fuentes de actualización; 2) la producción de objetos de enseñanza, que tiene relación con el trabajo inmediato en el aula y la integración curricular, y que define la existencia de un campo aplicado en las instituciones formadoras de maestros. A este respecto, Hargreaves et al. señalan que:

Las culturas de cooperación en el trabajo o comunidades profesionales tienen muchos componentes. Cuando los docentes tenían experiencias de esa índole, valoraban las oportunidades y el aliento que les proporcionaban, para llevar a cabo un buen número de iniciativas. La colaboración (...) estimulaba su creatividad (...), les daba la confianza que necesitaban para ensayar nuevas ideas (...) y les ofrecía una red de apoyo que les permitía perseverar cuando hallaban algún inconveniente (...). Trabajando juntos, el profesorado obtiene "apoyo emocional, y no te sientes solo, lo que te da mucha seguridad" (2001, p. 176).

Lo anterior nos permite tomar dos ejes para el trabajo colaborativo. Por un lado, el conocimiento y la reflexión de las prácticas pedagógicas y, por otro, la narración de dichas experiencias. Como dicen Connelly y Clandinin:

En primer lugar, necesitamos escuchar atentamente a los profesores y a otras personas dispuestas a aprender y a los relatos de sus vidas tanto dentro como fuera de clase. En segundo lugar, tenemos que contar nuestras propias historias en tanto que también nosotros vivimos nuestras propias [y colaborativas] vidas de investigadores y profesores. Nuestro propio trabajo, entonces, se convierte en un trabajo que consiste en aprender a contar y a vivir un nuevo relato de investigación en la enseñanza y en el aprendizaje que esté construido de forma colaborativa. Y lo que emerge de esta relación colaborativa son nuevas historias de los profesores y de quienes aprenden como creadores de currículos, historias que ofrecen nuevas posibilidades tanto para los investigadores y los profesores implicados como para aquellos que lean sus historias. Para el currículum, y quizás para otras ramas de la investigación educativa, se trata de un programa de investigación que da algo que hacer a los profesores de currículum (1995, p. 51).

El trabajo de colaboración entre pares académicos supone, pues, una actitud de parte de los maestros que hace de estos unos aprendices permanentes, al tiempo que constituye el medio de realización efectiva de la autonomía, bien sea en el caso individual de los maestros que dejarían de ser meros administradores de un currículo impuesto desde fuera, o bien en el caso de las instituciones, responsables ellas mismas de sus propios proyectos educativos. Tales supuestos llevarían al establecimiento de comunidades críticas de investigadores, cuyas fuentes son de orden teórico, político y empírico, interesadas no solo en la comprensión aislada de temas puntuales de investigación educativa, sino también en el planeamiento y la ejecución de acciones transformadoras, en "una espiral de ciclos de acción y reflexión" (Carr y Kemmis, 1988, p. 157). Para estos autores, los ciclos de reflexiónacción conducen a la conformación de grupos, pues se requiere la colaboración para revisar la praxis individual y las interacciones que a partir de ella establecen en las instituciones en las cuales trabajan.

La investigación-acción, en cuanto que se ocupa del mejoramiento de las prácticas, de los entendimientos y de las situaciones de carácter educativo, se basa necesariamente en un enfoque de la verdad y de la acción como socialmente construidas e incorporadas en la historia. En primer lugar es en sí misma un proceso histórico de transformación de prácticas, de entendimientos y de situaciones: tiene lugar en la historia y a través de ella. Todo estudio o proyecto de investigación-acción empieza con un patrón de prácticas y de entendimientos en una situación, y termina con otro distinto en el que algunas prácticas o algunos elementos de éstas serán continuos durante el proceso de mejoramiento, mientras que otros serán discontinuos (se han añadido nuevos elementos, otros han quedado abandonados, y unos terceros han experimentado tales o cuales transformaciones). De manera similar, los entendimientos también atraviesan un proceso de transformación histórica. Y las situaciones dentro de las cuales se conducen las prácticas habrán quedado transformadas en uno u otro sentido (p. 193).

No obstante, no todo trabajo en equipo es garantía de eficiencia y productividad, pues no pocas veces este se convierte en un refugio de superficialidad o de rutina burocrática. En palabras de Imbernón:

Hargreaves (1996) distingue entre colegialidad artificial y cultura colaborativa. La primera está caracterizada por una serie de procedimientos formales, específicos y burocráticos que pueden verse como iniciativas de asesoramiento entre iguales, de supervisión clínica, de planificación colectiva, que se realizan en espacios determinados y en reuniones formales especiales para tales cometidos. La colegialidad artificial puede consumir energías y tiempo necesarios para realizar contactos e 
intercambios más auténticos entre el profesorado, y con ello abortar procesos de mejora real. En cambio, las culturas colaborativas reales se caracterizan por presentar contactos más profundos, personales y duraderos o permanentes, que se realizan no únicamente en sesiones o reuniones concretas o con objetivos especiales, sino que se manifiestan en el trabajo diario del profesorado (1994, p. 106).

La colegialidad, en una cultura colaborativa real, se caracteriza por los vínculos que establecen los docentes en relación con las actividades cotidianas que se llevan a cabo a través de la práctica pedagógica. Nuestra búsqueda es contraria a esa colegialidad artificial, pues proponemos que el equipo docente sea una manera de acoger, desde la experiencia de sí y de los otros, teorizaciones, conceptualizaciones, aplicaciones, apropiaciones, innovaciones y prácticas, sobre la formación de maestros, las ciencias, la cultura, el arte, lo público, etc., en tanto referentes formativos. Esto es, que "la confluencia de perspectivas diferentes en el análisis" (Vilarruibias y Mauri, 2001, p. 29) de las situaciones, proyectos, propuestas y prácticas en la escuela normal superior, contribuya a la construcción de un escenario de trabajo colaborativo que dé cohesión social y pedagógica para trabajar en un ambiente de confianza.

\section{Formas de funcionamiento del equipo docente en las Escuelas Normales Superiores}

La experiencia del equipo docente, concebida intersubjetivamente, nos impone una noción de maestro no como sujeto solipsista y egoísta, sino en relación con los otros y capaz de acceder a la experiencia de sí. Por tanto, pensar al maestro unilateralmente desde la ciencia que enseña, o la infancia, o las necesidades sociales, económicas y políticas, es una reducción de las múltiples formas de existencia del maestro, que no está circunscrito al aula y a lo que exclusivamente pasa allí en su relación con los alumnos. El maestro debe construir vínculos con sus colegas, con el campo conceptual de la pedagogía, con la sociedad, con lo público, con la ciencia, con la cultura, con el arte, como referentes formativos, desde su condición de maestro.

Lo anterior nos aboca a una de las posibilidades del dispositivo formativo comprensivo: la superación del abismo que separa a los investigadores de los maestros, cuya relación es definida en el campo intelectual de la educación como productores (los intelectuales de la educación) y reproductores (los maestros), con la mediación de un campo de recontextualización. El equipo docente, estructurado en núcleos, es la estrategia diseñada para superar ese abismo y abrir efectivos canales de comunicación con las comunidades científicas y académicas.

De este modo, pueden distinguirse tres niveles en la organización del trabajo académico:

1. Un equipo docente, conformado alrededor de un proyecto general de investigación.

2. Los núcleos (o colegios académicos, o como se les quiera llamar) que reúnan a los especialistas de las diversas disciplinas del conocimiento. El núcleo es un espacio de múltiples relaciones en la escuela normal superior, una de las cuales es la formación a través de la autoformación y la conformación. Ambas interactúan para configurar el saber del maestro en torno a la práctica, la experiencia, la experimentación, la innovación y la investigación en el aula, y son despliegues del campo aplicado en la escuela normal.

3. Los proyectos de aula articulados a la dinámica del campo aplicado.

Con estas formas de funcionamiento, estamos cerca de lo que Hargreaves (1996) señala como cultura de la colaboración, y difiere de otras formas de trabajo, como la balcanización o la colegialidad restringida, en la que los intereses individuales o las tareas asignadas por las administraciones de las Escuelas Normales son la base del encuentro y el trabajo conjunto. La cultura de la colaboración se centra en la constitución de una meta común, con las miras puestas en las mismas intencionalidades, y con los proyectos conjuntos orientados por un interés general. Esta cultura no se logra de un momento a otro, como indican Delaire y Ordronneau:

Entre la necesidad de las propias dificultades y la formación de un equipo a veces transcurre mucho tiempo, el tiempo de la maduración. Se pasa por la fase del intercambio de recetas y progresivamente la célula que era al principio binaria, se amplía, hasta que un día toma cuerpo un proyecto más preciso entre colegas de un mismo curso o de una misma materia o de dos cursos o centros. Surge entonces la decisión de crear un equipo: insistimos en el desarrollo más frecuente del proceso. No se hace equipo de un día para otro si no se ha preparado. No sirve de nada forzar a los colegas reticentes como si se les quisiera hacer entrar en una secta. Que los que deseen poner en marcha un equipo lo hagan funcionar; como es natural, la idea positiva que se deriva de la experiencia será más convincente que la presión, que produce 
efectos generalmente contrarios a los esperados (1991, p. 75).

Se trata, por tanto, de constituir un equipo docente donde la experiencia de sí y del otro haga visible el cuerpo del maestro, su propio rostro, en los procesos de formación inicial y de formación permanente, en las instituciones formadoras de docentes.

En tal sentido, la propuesta consiste en poner a disposición de una institución formadora de maestros unas instancias donde la experiencia de sí del maestro y su relación con los otros no esté determinada exclusivamente por factores externos, sean estos la infancia, las necesidades sociales y políticas, los nuevos contextos informáticos, los avances de las ciencias cognitivas, entre otros, y que permitan la creación de nuevas temporalidades y espacialidades en la institución, que coadyuven a la constitución del equipo docente en la colegialidad, la cooperación y la autogestión intelectual e investigativa.

Permitir que los profesores dispongan de tiempo para trabajar juntos durante la jornada escolar ayuda a mejorar la calidad del currículum y de la enseñanza que imparten a sus alumnos. Las limitaciones y restricciones en el tiempo de preparación perjudican la capacidad de los docentes para innovar de manera eficaz y rebajan el nivel de lo que son capaces de preparar para sus clases (Hargreaves et al., 2001, p. 181).

Nuestra comprensión del equipo docente reivindica una propuesta que permite, desde las posibilidades de una experiencia narrativa de sí mismo y de los otros, otras perspectivas del análisis de la problemática del maestro y de su formación. Por tanto, la práctica pedagógica debe ser concebida como una experiencia ${ }^{7}$, como algo que nos pasa, que pasa en las instituciones educativas. De este modo, será posible comprender el movimiento interior de las Escuelas Normales, a la vez que identificar los factores necesarios de intervenir a través del equipo docente.

No parece especialmente revolucionario decir que es importante intentar entender cómo funcionan los profesores y las aulas antes de repartir recomendaciones para el cambio. Sin embargo, gran parte de lo que se ha sugerido a los profesores y administradores de escuela se ha propuesto de forma independiente del contexto y, a menudo, por personas que desconocían las prácticas que intentaban mejorar. Si la indagación cualitativa en educación trata de algo, es

7 Eisner indica que "la experiencia tiene su génesis en nuestra transacción con las cualidades que constituyen nuestro entorno. Entiendo por cualidades esos rasgos de nuestro entorno que podemos experimentar a través de nuestros sentidos" (1998, p. 35) sobre cómo intentar comprender lo que los profesores y los niños hacen en los grupos:

Que trabajan. Para alcanzar este propósito -y existen otros propósitos igualmente importantes para la indagación cualitativa-es necesario prestar atención a las escuelas y las aulas por las que nos preocupamos, observarlas y utilizar lo que vemos como fuente de interpretación y valoración (Eisner, 1998, pp. 27-28).

Por tanto, se busca instalar al maestro en múltiples relaciones, recogidas en una subjetividad narrativa postulada como cuerpo y en vínculo con los otros, que permita desde su formación inicial ser visible para sí. Esta línea de trabajo es afín a los propósitos de la llamada investigación narrativa, que según Greene evita que "el profesor sea tratado como si no tuviera una vida propia, como si no tuviera un cuerpo, un lenguaje, una historia o una interioridad y parecen presuponer "un hombre dentro del hombre" (1995, p. 82).

En otras palabras, la propuesta apunta a que antes o al mismo tiempo de entrar en relación con el mundo, con sus prácticas sociales, es necesario que el maestro tenga un espacio, un lugar desde donde pensar, hablar y actuar en el mundo del saber y el conocimiento. Es este el problema que el maestro debe resolver, es un punto que se debe comprender muy bien, porque significa defender una metodología de construcción de la escuela normal superior, de adentro hacia afuera.

La tarea es apropiarse del saber, del conocimiento, etc., y desde ahí entrar en relación con el mundo. Ese conocimiento no puede ser otro que aquellas cosas en las que cotidianamente el maestro trabaja: la enseñanza, el aprendizaje, la formación. Este enfoque evita los externalismos, porque es desde el interior de una institución fortalecida donde se inicia la construcción de esa institución, y ello implica una nueva forma de relacionarse con el saber y el conocimiento, los cuales permiten el acceso a las necesidades sociales, políticas y económicas. Pero si no partimos de la construcción de esa casa desde la cual podamos hablar el lenguaje de aquello con que trabajamos permanentemente, nos vamos a disolver en esas demandas de la sociedad, y vamos a terminar convertidos en un trabajo de tipo asistencial.

\section{Una primera finalización}

Los primeros resultados de esta investigación nos muestran lo siguiente:

- El equipo docente produce efectos de ruptura sobre el asignaturismo, el tiempo técnico racional 
y el encierro del dispositivo anexa en las escuelas normales superiores de Antioquia, por cuanto posibilita reordenamientos espaciales, temporales e intersubjetivos que inciden en la apropiación del dispositivo formativo comprensivo, la construcción de los núcleos, los proyectos de investigación y los planes de estudio.

- El equipo docente permite la reconstrucción de la identidad pedagógica del maestro a través de la experiencia narrativa de sí mismo y de los otros, manifiesta en la escritura a través de la autobiografía pedagógica.

- El equipo docente posibilita recuperar el relato como instancia constitutiva de una identidad narrativa a través de la voz como aquello que da sentido, donde reside el individuo y le permite participar en una comunidad.

- El equipo docente contribuye a reconstruir la voz del maestro desde la perspectiva de las relaciones con el sentido de su experiencia, con el lenguaje y con el otro.

- El equipo docente transforma las narraciones y relatos que el maestro realiza sobre su práctica pedagógica, dándole la posibilidad de reflexionar su práctica docente cotidiana y, en consecuencia, desarrollar reflexiones que conmueven su intimidad, cuestionan, interrogan y transforman.

\section{Referencias biliográficas}

Carr, W. y Kemmis, S. (1988). Teoría crítica de la enseñanza. Barcelona: Martínez Roca.

Connelly, M. y Clandinin, J. (1995). Relatos de experiencia e investigación narrativa. En Larrosa, J. et al., Déjame que te cuente. Ensayos sobre narrativa y educación. Barcelona: Laertes.

Contreras, J. (1996). Teoría y práctica docente. Cuadernos de Pedagogía, 253, 92-100.

Delaire, G. y Ordronneau, H. (1991). Los equipos docentes. Formación y funcionamiento. Madrid: Narcea.
Echeverri, J. (1996). Premisas conceptuales del dispositivo formativo comprensivo, Educación y Pedagogía, Medellín, Universidad de Antioquia, No. 16, 71-105.

Echeverri, J. (2000-2003). “Apropiación pedagógica del campo intelectual de la educación para la construcción de un modelo comprensivo para la formación de docentes", proyecto de investigación financiado por Colciencias, la Universidad de Antioquia y la Secretaría de Educación y Cultura de Antioquia.

Echeverri, J. (2008). Aciforma: los seminarios permanente como el mayor aporte a la fundación de las Escuelas Normales Superiores, 1999-2000. A(u)las, Revista Pedagógica, Escuela Normal Superior María Auxiliadora, 5, 115-137.

Eisner, E. (1998). El ojo ilustrado. Indagación cualitativa y mejora de la práctica educativa. Barcelona: Paidós.

Giroux, H. (1996). Placeres inquietantes. Barcelona: Paidós.

Greene, M. (1995). El profesor como extranjero. En Larrosa, J. et al., Déjame que te cuente. Ensayos sobre narrativa y educación (pp. 81-130). Barcelona: Laertes.

Hargreaves, A. (1996). Profesorado, cultura, posmodernidad. Cambian los tiempos, cambia el profesorado. Madrid: Morata.

Hargreaves, A., Earl, L., Moore, S. y Manning, S. (2001). Aprender a cambiar. La enseñanza más allá de las materias y los niveles. Barcelona: Octaedro.

Imbernón, F. (1994). La formación y el desarrollo profesional del profesorado. Barcelona: Graó.

Larrosa, J. (1995). Tecnologías del yo y educación. (Notas sobre la construcción y la mediación pedagógica de la experiencia de sí). En Larrosa, J. (ed.), Escuela, poder y subjetivación (pp. 255-329). Madrid: Ediciones de La Piqueta.

Vilarruibias, P. y Mauri, T. (2001). Todo lo que se puede formar en la formación de centros. En La formación del profesorado. Proyectos de formación en centros educativos (pp. 21-34). Barcelona: Grao. 


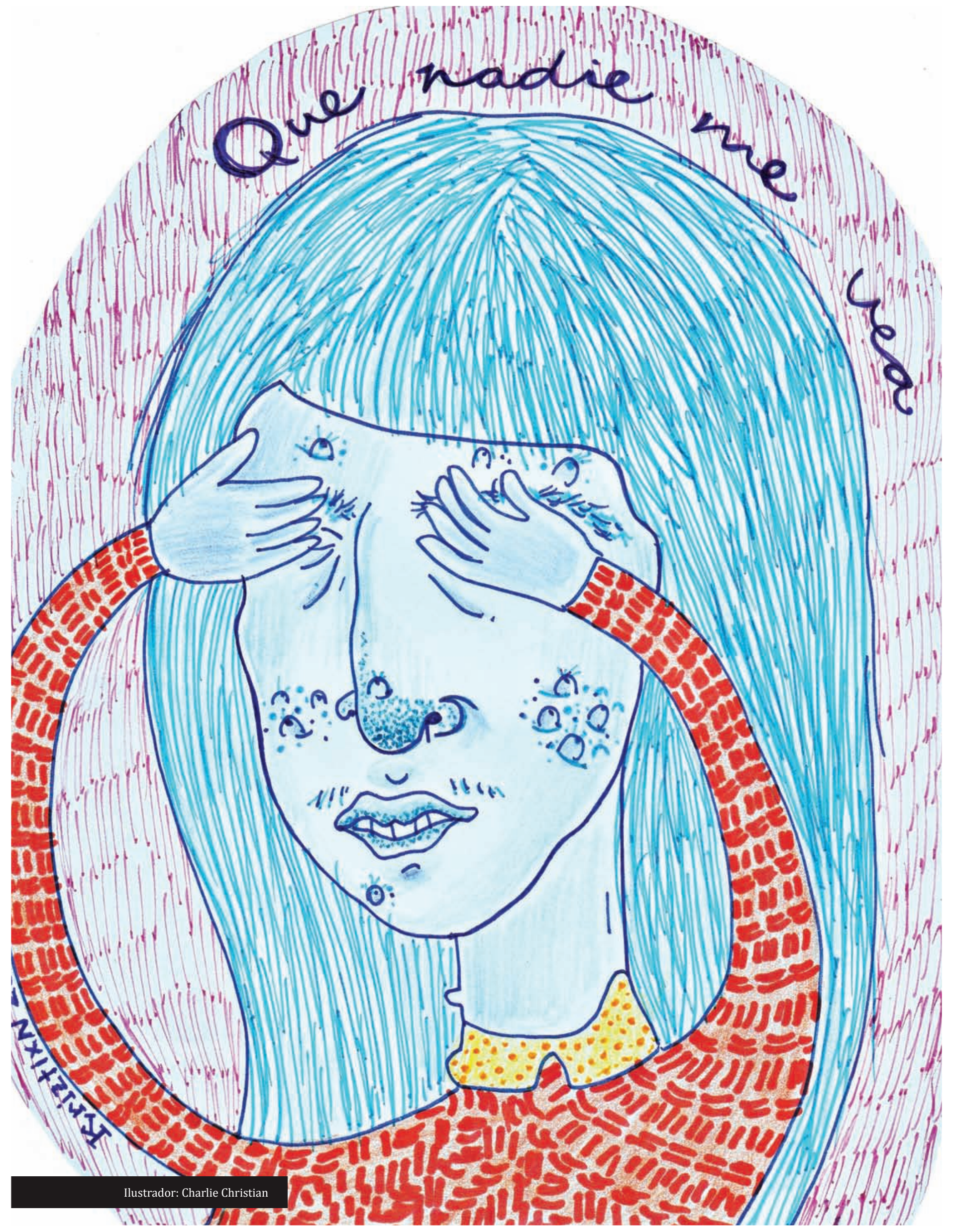

